\title{
Paper REG-360
}

\section{Multiple Input Design for Real-Time Parameter Estimation in the Frequency Domain}

Eugene A. Morelli

NASA Langley Research Center

Hampton, Virginia USA

$13^{\text {th }}$ IFAC Conference on System Identification August 27-29, 2003 / Rotterdam, The Netherlands 


\title{
MULTIPLE INPUT DESIGN FOR REAL-TIME PARAMETER ESTIMATION IN THE FREQUENCY DOMAIN
}

\author{
Eugene A. Morelli \\ NASA Langley Research Center \\ Hampton, Virginia USA
}

\begin{abstract}
A method for designing multiple inputs for real-time dynamic system identification in the frequency domain was developed and demonstrated. The designed inputs are mutually orthogonal in both the time and frequency domains, with reduced peak factors to provide good information content for relatively small amplitude excursions. The inputs are designed for selected frequency ranges, and therefore do not require a priori models. The experiment design approach was applied to identify linear dynamic models for the F-15 ACTIVE aircraft, which has multiple control effectors.

Copyright (C) 2003 IFAC
\end{abstract}

Keywords: experiment design, parameter estimation, frequency domain, aircraft system identification

\section{INTRODUCTION}

Frequency domain techniques have been used successfully to identify dynamic models for aircraft, including cases where the aircraft was open-loop unstable (Schkolnik, et al., 1995; Morelli, 2002). Recent work has indicated that real-time parameter estimation for aircraft dynamic models can be done effectively using a recursive chirp- $Z$ transform for a selected frequency band, then employing equation-error parameter estimation in the frequency domain (Morelli, 2000). Advantages of this approach include robustness to measurement biases, noise, and infrequent dropouts in the time-domain data, enhanced signal-to-noise ratio in the frequency domain, accurate parameter estimates and error bounds in real time, with very low computational and memory requirements.

Good experiment design for dynamic modelling in the frequency domain requires that excitation inputs to the dynamic system contain a variety of frequencies. At the same time, the excitation must be such that the amplitudes of the dynamic system responses are not too large, so that the model structure assumption, which is typically linear, is not violated. For the case of real-time parameter estimation on aircraft, inputs are preferably small enough so that the pilot cannot distinguish the aircraft response to excitation inputs from a typical aircraft response to turbulence. Modern aircraft have multiple control effectors, so it would be advantageous if the excitation for frequency domain identification could be applied to multiple control effectors simultaneously, so that the amount of time required to collect data for dynamic modelling could be reduced.

Previous work (Schroeder, 1970) has shown that a phase-shifted sum of sinusoids, called the Schroeder sweep, provides an input with good frequency content and low peak factor. The peak factor is a measure of the ratio of maximum input amplitude to input energy. Inputs with low peak factors are efficient in the sense of providing good frequency content without large amplitudes in the time domain. Comparisons of the Schroeder sweep with conventional linear and logarithmic frequency sweep inputs have indicated that the Schroeder sweep is generally the superior input for frequency domain dynamic model identification (Young and Patton, 1990). The Schroeder sweep has been used successfully in other practical system identification problems (Flower, et al., 1978; Bosworth and Burken, 1997).

This paper describes an extension of the Schroeder sweep input design method to multiple input design with optimised peak factors for real-time parameter estimation. The designed inputs are mutually orthogonal in both the time domain and the frequency domain, and are formulated as perturbation inputs. The only a priori information required for the multiple input design is an estimate of the approximate frequency band for the system dynamics and approximate relative control effectiveness for proper relative scaling of the input amplitudes. Investigations were conducted to determine signal-to-noise ratios necessary to achieve good parameter estimation results from the real-time 
parameter estimator. This information is necessary so that the input amplitude scaling can adapt to different aircraft dynamics throughout the flight envelope.

The multiple input design technique was applied to a lateral / directional linear simulation for the F-15 ACTIVE aircraft, which includes four control effectors. Real-time parameter estimation in the frequency domain (Morelli, 2000) was used to estimate the dynamic model parameters. Parameter estimation results were compiled and analysed.

The next section describes the multiple input design procedure. Following this, real-time parameter estimation results from applying the designed inputs to the F-15 ACTIVE simulation are presented and discussed.

\section{MULTIPLE INPUT DESIGN}

Each input to the aircraft control surfaces is comprised of a set of summed harmonic sinusoids with individual phase lags. Each input $\boldsymbol{u}_{j}$, applied to

the $j^{\text {th }}$ control surface, takes the form

$$
\boldsymbol{u}_{j}=\sum_{k \in\{1,2, \ldots, M\}} A_{k} \cos \left(\frac{2 \pi k t}{T}+\phi_{k}\right)
$$

where $M$ is the total number of available harmonically-related frequencies, $T$ is the time length of the excitation, and the $\phi_{k}$ are phase angles to be chosen for each of the harmonic components to produce a low peak factor $P F$, defined by

$$
P F\left(u_{j}\right)=\frac{\left[\max \left(u_{j}\right)-\min \left(u_{j}\right)\right] / 2}{\sqrt{\left(u_{j}^{T} u_{j}\right) / N}}
$$

or

$$
P F\left(u_{j}\right)=\frac{\left[\max \left(u_{j}\right)-\min \left(u_{j}\right)\right]}{2 r m s\left(u_{j}\right)}=\frac{\left\|u_{j}\right\|_{\infty}}{\left\|u_{j}\right\|_{2}}
$$

where the last equality only holds when $\boldsymbol{u}_{j}$ oscillates symmetrically about zero. In the literature, the quantity $\left\|u_{j}\right\|_{\infty} /\left\|u_{j}\right\|_{2}$ is called the crest factor. A single sinusoidal component from the summation in Eq. (1) has $P F=\sqrt{2}$, so the relative peak factor $R P F$, defined by

$$
R P F\left(u_{j}\right)=\frac{\left[\max \left(u_{j}\right)-\min \left(u_{j}\right)\right]}{2 \sqrt{2} r m s\left(u_{j}\right)}=\frac{P F\left(u_{j}\right)}{\sqrt{2}}
$$

quantifies the peak factor relative to that of a single sinusoid. For a single sinusoid, $R P F$ equals 1 . The relative peak factor is a measure of efficiency of a input for parameter estimation purposes, in terms of the amplitude range of the signal divided by a measure of the signal energy. Lower relative peak factors are more desirable for parameter estimation, where the objective is to excite the system without driving it too far away from the nominal operating point, so that model structure assumptions are not violated.

The integers $k$ specifying the frequencies for the $j^{\text {th }}$ input $u_{j}$ are unique to that input, but the integers $k$ used in the summation in Eq. (1) are not necessarily consecutive, as will be explained below. The objective for the experiment design is to excite the aircraft dynamics in a short time period by moving multiple control surfaces simultaneously. Since more than one surface is being moved, it is advantageous for the modelling if the $u_{j}$ vectors applied to each control surface are mutually orthogonal. This helps the parameter estimation by completely de-correlating the inputs to the aircraft, which improves the accuracy of control effectiveness estimates. It is possible to make the $u_{j}$ mutually orthogonal in both the time and frequency domains, using inputs designed for low relative peak factor, as will be shown next. This gives the analyst the flexibility to use time domain or frequency domain parameter estimation methods, while retaining the desirable feature of mutually orthogonal inputs in either domain.

In the time domain, a signal composed of a sum of sinusoids is orthogonal to any other sum of sinusoids with harmonically-related frequencies, regardless of the constant phase shift of each sinusoidal component contained in the signals. For example, if two inputs each contain a single, distinct, harmonically-related sinusoidal component,

$$
u_{1}=\cos \left(\frac{2 \pi t}{T}+\phi_{1}\right) \quad u_{2}=\cos \left(\frac{2 \pi 4 t}{T}+\phi_{2}\right)
$$

then the inner product of these inputs, using the discrete-time notation $t_{i}=i \Delta t$ and $T=(N-1) \Delta t$, is

$$
\begin{gathered}
u_{1}^{T} u_{2}=\sum_{i=0}^{N-1} \cos \left(\frac{2 \pi t_{i}}{T}+\phi_{1}\right) \cos \left(\frac{2 \pi 4 t_{i}}{T}+\phi_{2}\right) \\
=\sum_{i=0}^{N-1}\left[\cos \left(\frac{2 \pi t_{i}}{T}\right) \cos \left(\phi_{1}\right)-\sin \left(\frac{2 \pi t_{i}}{T}\right) \sin \left(\phi_{1}\right)\right] \\
\times\left[\cos \left(\frac{2 \pi 4 t_{i}}{T}\right) \cos \left(\phi_{2}\right)-\sin \left(\frac{2 \pi 4 t_{i}}{T}\right) \sin \left(\phi_{2}\right)\right]
\end{gathered}
$$

$=0$

The sine and cosine of the constant phase angles $\phi_{1}$ and $\phi_{2}$ are constants, so the summation equals zero because of the orthogonality of harmonically-related sinusoids. For more than one sinusoidal component in each input, the analysis is similar. So, the inputs assembled as in Eq. (1) are orthogonal in the time domain. 
If the frequency indices $k$ that are selected for each input $\boldsymbol{u}_{j}$ are distinct from those chosen for the other inputs, then the frequency content of each $u_{j}$ consists of distinct spectral lines in the frequency domain, since the summation in Eq. (1) is a cosine series with harmonically-related frequencies. Therefore, the vectors of Fourier transforms for the inputs as a function of frequency have inner products equal to zero. In this sense, the inputs are also mutually orthogonal in the frequency domain.

The multiple input design procedure is as follows:

1. Select the time period $T$ for the excitation, which determines the smallest harmonic frequency resolution $\Delta f=1 / T$ and the limit on the minimum frequency $f_{\min } \geq 2 / T$.

2. Select the frequency band of the dynamic system for the excitation frequencies, $\left[f_{\min }, f_{\max }\right] \mathrm{Hz}$. This corresponds to the frequency band where the expected dynamic response of the system will occur. The frequencies are equally spaced by $\Delta f$ on the interval $\left[f_{\min }, f_{\max }\right]$. The total number of frequencies $M=f i x\left\{\left(f_{\max }-f_{\min }\right) / \Delta f\right\}+1$, where fix indicates rounding to the nearest integer toward zero.

3. Assign approximately an equal number of indices $k$ from the set $\{1,2, \ldots, M\}$ to each input by alternating each consecutive frequency among the multiple inputs. This approach produces lower relative peak factors for the individual inputs, and also ensures that each input has frequency content distributed evenly across the frequency band $\left[f_{\min }, f_{\max }\right]$. Different assignments of the frequency indices could be made for other reasons. For example, lower frequency indices might be assigned to an input that is known to effectively excite a low frequency mode, or particular frequency indices might be omitted to avoid exciting an undesirable structural response. It was found empirically that if the set of selected indices $k$ for a particular input consisted of an integer greater than 1 , with 2 or 3 multiples of that integer (e.g., $k=2,4,6$, or $k=5,10$, 15,20 ), the phase angles could be optimised (in step 5) so that the relative peak factor for that input was very close to 1 , and sometimes less than 1 . Each frequency index can be assigned to only one of the inputs, to preserve mutual orthogonality of the inputs in both the time and frequency domains.

4. Generate the input $u_{j}$ for each of the $m$ controls, using Eq. (1) and computing the starting values for the phase angles $\phi_{k}$ according to method described by Schroeder (1970), assuming a uniform power spectrum.
5. Use a simplex optimisation algorithm (Press, et $a l ., 1992)$ to adjust the $\phi_{k}$ for each $u_{j}$ to achieve minimum relative peak factor for that input. The optimisation algorithm does not require gradients.

6. For each input, do a one-dimensional search to find a constant time offset for the components of each input $u_{j}$, so that the input begins and ends at zero amplitude. This is equivalent to sliding the input along the time axis until a zero crossing is placed at the origin of the time axis. The appropriate phase shift is added to each sinusoidal component phase shift $\phi_{k}$. Note that to implement a constant time shift to all the components, the phase offset for each component will be different, because each component has a different frequency. Since the components of each $u_{j}$ are harmonics of the base frequency with period $T$, if all the component phase angles $\phi_{k}$ are shifted so that the initial value of the input is zero, then the final value of the input will also be zero. The power spectrum, input orthogonality, and relative peak factor are all unaffected.

7. Return to step 5 until the relative peak factor reaches a pre-defined goal value or until a maximum number of iterations is reached. For this work, the relative peak factor goal was set at 1.01 and the maximum number of iterations was set to 50 .

Although there are methods for optimising the frequency spectrum of inputs for parameter estimation, all of them require considerable computation, along with some knowledge of the system dynamics, usually in terms of a nominal model with parameter values. In the current application, there is no use for such methods, because the intent is to identify the aircraft dynamics in real time, for various flight conditions throughout the flight envelope, and for arbitrary failure conditions, to enable control reconfiguration. Under these circumstances, computing an optimised frequency spectrum does not make sense. Instead, the frequency spectra for all inputs were defined to be flat across the selected frequency band, so that the aircraft dynamics would be sufficiently excited, regardless of where the modal frequencies happen to be located within the frequency band.

Step 6 of the input design procedure ensures that each designed input will be a perturbation, so that the designed input can be added to whatever constant value the control may have for another reason, e.g., trim or manoeuvring. The iteration helps to reduce the relative peak factors by adding perturbations to the phase angles $\phi_{k}$ (from the phase changes added to make the input start and end at zero), which helps the optimisation algorithm solve this non-convex optimisation problem.

Figure 1 shows the relative peak factor as a function of phase angles for two components in the summation of Eq. (1), using $T=15 \mathrm{sec}$, unit amplitudes, and 
frequency indices $k=2$ and $k=4$. It is clear from the figure that this optimisation problem is not convex, and also that there are several phase angle solutions which are equally good, or nearly so. The data used to make the plot in Figure 1 can be used for a global exhaustive search for the minimum peak factor in this simple case. To generate this data, the relative peak factors were computed for values of the phase angles on a 2-dimensional grid with intervals of $0.0175 \mathrm{rad}$ ( $1 \mathrm{deg})$, over the range $[0, \pi] \mathrm{rad}([0,180] \mathrm{deg})$. The minimum relative peak factor of all these computed values was found to be 1.102 . Using the technique described above to optimise the phase angles, the relative peak factor achieved was 1.106 . This demonstrated that the technique developed here found an input with peak factor very close to the global optimum, in this simple case. The same exercise was then repeated for three harmonic components, with frequency indices $k=2,4$, and 6 , and the same phase angle intervals, in three dimensions. In this case, the global exhaustive search gave a minimum relative peak factor equal to 1.002 , while the optimisation technique described above produced an input with relative peak factor equal to 1.003. These investigations suggest that the procedure described here does an excellent job of designing inputs with very low peak factor.

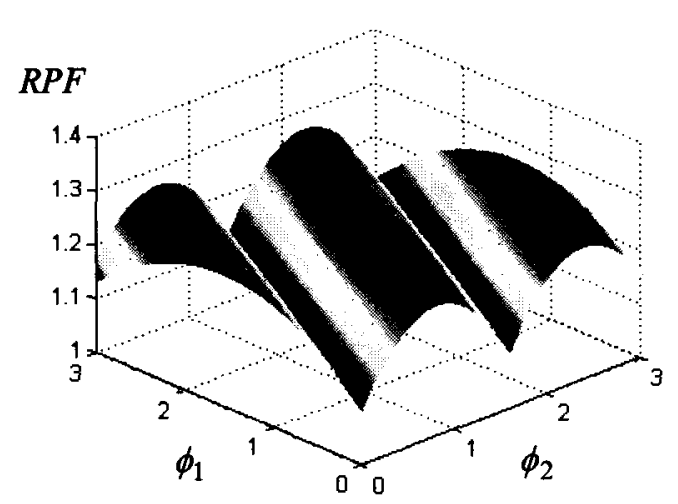

Fig. 1. Peak Factors for a Two-Component Input

\section{RESULTS}

For small perturbation motions about a reference condition, airplane dynamics can be described by the following linear model equations:

$$
\begin{aligned}
\dot{x}(t) & =A x(t)+B u(t) \quad x(0)=x_{o} \\
y(t) & =C x(t)+D u(t) \\
z_{i} & =y_{i}+v_{i} \quad i=0,1,2, \ldots, N-1
\end{aligned}
$$

Matrices $A, B, C$, and $D$ in Eqs. (5) and (6) contain stability and control parameters, which are to be estimated from flight data.

For the linearized lateral/directional dynamics of the F-15 ACTIVE aircraft, the state vector $x$ and input vector $\mathbf{u}$ in Eq. (1) are

$$
\boldsymbol{x}=\left[\begin{array}{llll}
\beta & p & \boldsymbol{r} & \phi
\end{array}\right]^{T} \quad \boldsymbol{u}=\left[\begin{array}{llll}
\delta_{a} & \delta_{r} & \delta_{d c} & \delta_{d s}
\end{array}\right]^{T}
$$

The output vector is:

$$
\boldsymbol{y}=\left[\begin{array}{lllll}
\beta & \boldsymbol{p} & \boldsymbol{r} & \phi & a_{y}
\end{array}\right]^{T}
$$

System matrices containing model parameters are:

$$
\begin{gathered}
\boldsymbol{A}=\left[\begin{array}{ccccc}
Y_{\beta} & \sin \alpha_{o} & -\cos \alpha_{o} & g \cos \theta_{o} / V_{o} \\
L_{\beta} & L_{p} & L_{r} & 0 \\
N_{\beta} & N_{p} & N_{r} & 0 \\
0 & 1 & \tan \theta_{o} & 0
\end{array}\right] \\
\boldsymbol{B}=\left[\begin{array}{ccccc}
0 & Y_{\delta_{r}} & Y_{\delta_{\star}} & Y_{\delta_{\alpha}} \\
L_{\delta_{\alpha}} & L_{\delta_{r}} & L_{\delta_{\alpha}} & L_{\delta_{\star}} \\
0 & N_{\delta_{r}} & N_{\delta_{\alpha}} & N_{\delta_{\alpha}} \\
0 & 0 & 0 & 0
\end{array}\right] \\
\boldsymbol{C}=\left[\begin{array}{cccc}
1 & 0 & 0 & 0 \\
0 & 1 & 0 & 0 \\
0 & 0 & 1 & 0 \\
0 & 0 & 0 & 1 \\
\frac{V_{o}}{g} Y_{\beta} & 0 & 0 & 0
\end{array}\right] \quad D=\frac{V_{o}}{g}\left[\begin{array}{cccc}
0 & 0 & 0 & 0 \\
0 & 0 & 0 & 0 \\
0 & 0 & 0 & 0 \\
0 & 0 & 0 & 0 \\
0 & Y_{\delta_{r}} & Y_{\delta_{\alpha}} & Y_{\delta_{\alpha}}
\end{array}\right]
\end{gathered}
$$

Figure 2 shows the NASA F-15 ACTIVE aircraft (Doane, et al., 1994). The values of the parameters in the model that were used to generate the simulated data are given in column 2 of Table 1.

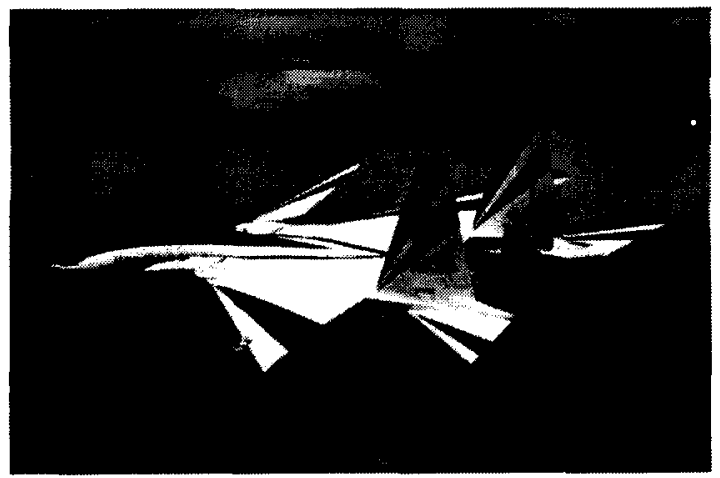

Fig. 2. F-15 ACTIVE Aircraft

The multiple input design for the F-15 ACTIVE aircraft lateral/directional dynamics includes four control effectors: aileron, rudder, differential canard, and differential stabilator. Figure 3 shows the time histories of the multiple input design for an $18 \mathrm{sec}$ manoeuvre, which includes 1 sec for steady trim flight at the start, and $2 \mathrm{sec}$ for free response at the end. The frequency range for the input design was chosen as $[0.2,1.4] \mathrm{Hz}$. The inputs shown are mutually orthogonal in both the time domain and the frequency domain. These inputs were designed using a flat power spectrum with $T=15$ sec. Table 2 contains the amplitudes, frequency indices, and the $R P F$ achieved for the $15 \mathrm{sec}$ inputs shown in Figure 3. All of the designed inputs achieved an extremely low relative peak factor close to 1 . 
Figure 4 shows the simulated responses, which include added noise equal to time domain residual sequences from flight test data analysis and modelling at the same flight condition for the real F-15 ACTIVE airplane. This ensures that a realistic noise environment was used in the investigations concerning the effectiveness of the multiple input design. Real-time parameter estimation in the frequency domain (Morelli, 2000) was used to estimate the parameters. The frequencies chosen for the recursive chirp- $Z$ transform were evenly spaced in $0.01 \mathrm{~Hz}$ increments on the interval $[0.11,1.5] \mathrm{Hz}$. This frequency band must include all of the input design frequency band, so that the important control surface information in the frequency domain is included in the parameter estimation. Table 1 contains the parameter estimation results for this case, using only the final values of the real-time parameter estimates and standard errors. The parameter estimation was started with no $a$ priori information for the parameter estimates. The results in Table 1 show that the model parameters are estimated very accurately, and that the computed standard errors are representative of the true error in the parameter estimates.

The mean signal-to-noise ratio for the outputs shown in Figure 4 was 30. It is of interest to obtain good parameter estimation results with the lowest possible signal-to-noise ratio on the outputs, so that the aircraft and pilot are disturbed as little as possible by the activity on the control surfaces. Figure 5 shows the effect of output signal-to-noise ratio on the mean of the parameter estimate errors. The data for this plot was generated by reducing the input amplitudes uniformly using the same input forms shown in Figure 3, generating new simulated outputs, then adding the same noise sequences from flight test data used before, so that the output signal-to-noise ratio decreased. For output signal-to-noise ratio equal to 10 , the input amplitudes were $0.24 \mathrm{deg}$, and the mean parameter estimate error was 2.7 percent. This choice of input amplitudes produced excellent parameter estimate accuracy, while keeping the root-meansquare value of the lateral acceleration $a_{y}$ below $0.02 \mathrm{~g}$, which is less than $0.05 \mathrm{~g}$ that is typical for moderate turbulence.

\section{CONCLUDING REMARKS}

A multiple input design technique for real-time frequency domain parameter estimation was described and demonstrated. The technique can be used to design multiple inputs that are mutually orthogonal in both the time and frequency domains, with very low peak factors. Input energy over a selectable range of frequencies can be efficiently injected into the dynamical system by virtue of low peak factors and the ability to move inputs simultaneously, since the inputs are mutually orthogonal. These features make the inputs attractive for real-time parameter estimation application, where the excitation must have short duration and low output response amplitudes, and the dynamic response and modal frequencies will change because of flight condition changes or failure conditions.

To use this input design technique effectively in flight, the input forms could remain fixed, and a simple feedback could be used to scale the input amplitudes so that the output signal-to-noise ratios are near a chosen acceptable value. The excitation time $T$ should be minimized, and this will be impacted by the output signal-to-noise ratios. In addition, the F-15 ACTIVE airplane has a stability augmentation system, which will distort the input forms designed for parameter estimation. This affects the required input amplitudes, and degrades peak factors and input orthogonality. Further work is required to address these practical considerations.

The multiple input design technique described here can be applied to other problems as well, because there is no dependence on a priori models or information, other than the expected frequency range of the dynamic modes. Specific spectral weighting using the component amplitudes can be introduced, if warranted by $a$ priori information.

\section{REFERENCES}

Bosworth, J.T. and J.J. Burken (1997) "Tailored Excitation for Multivariable Stability-Margin Measurement Applied to the X-31A Nonlinear Simulation," AIAA 94-3361.

Doane, P., R. Bursey, and G. Schkolnik (1994) "F-15 ACTIVE: A Flexible Propulsion Integration Testbed," AIAA 94-3361.

Flower, J.O., G.F. Knott, and S.G. Forge (1978) "Application of Schroeder-phased Harmonic Signals to Practical Identification," Measurement and Control, 11, No. 2, pp. 69-73.

Morelli, E.A. (2000) "Real-Time Parameter Estimation in the Frequency Domain," Journal of Guidance, Control, and Dynamics, 23, No. 5, pp. 812-818.

Morelli, E.A. (2002) "System IDentification Programs for AirCraft (SIDPAC)," AIAA-20024704.

Press, W.H., S.A. Teukolsky, W.T. Vettering, and B.R. Flannery (1992) Numerical Recipes in FORTRAN: The Art of Scientific Computing, $2^{\text {nd }}$ Ed., Cambridge University Press, New York, NY, Chapter 10.

Schroeder, M.R. (1970) "Synthesis of Low-PeakFactor Signals and Binary Sequences with Low Autocorrelation," IEEE Transactions on Information Theory, pp. 85-89. 
Schkolnik, G.S., J.S. Orme, and M.A. Hreha (1995) "Flight Test Validation of a Frequency-Based System Identification Method on an F-15 Aircraft," NASA TM 4704.

Young, P. and R.J. Patton (1990) "Comparison of Test Signals for Aircraft Frequency Domain Identification," Joumal of Guidance, 13, No. 3, pp. $430-438$.
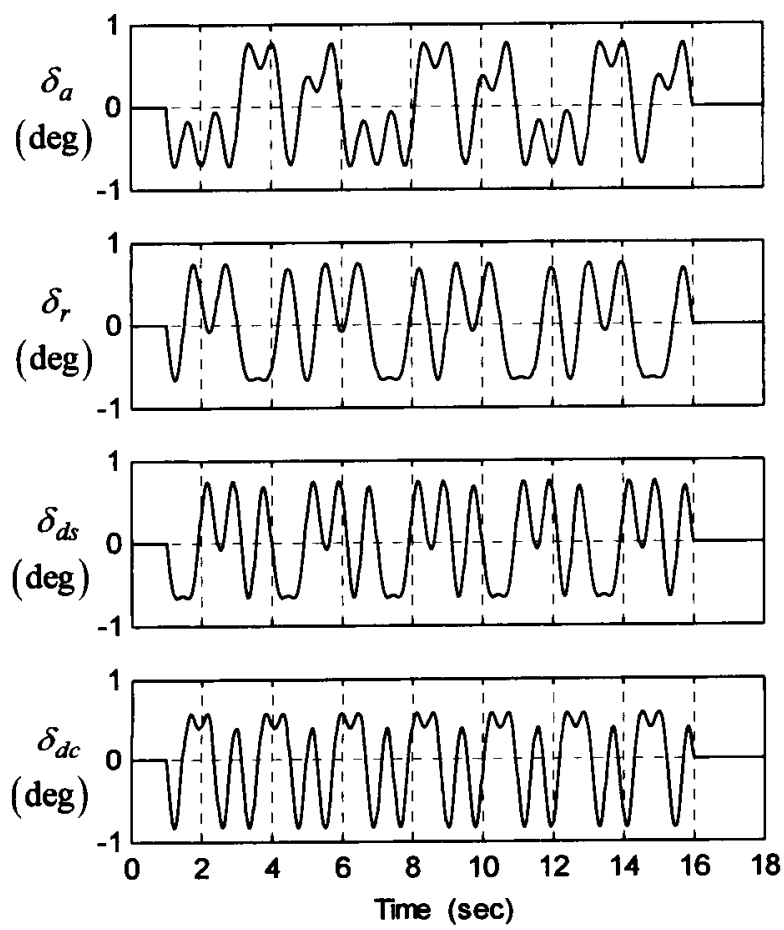

Fig. 3. Multiple Input Design
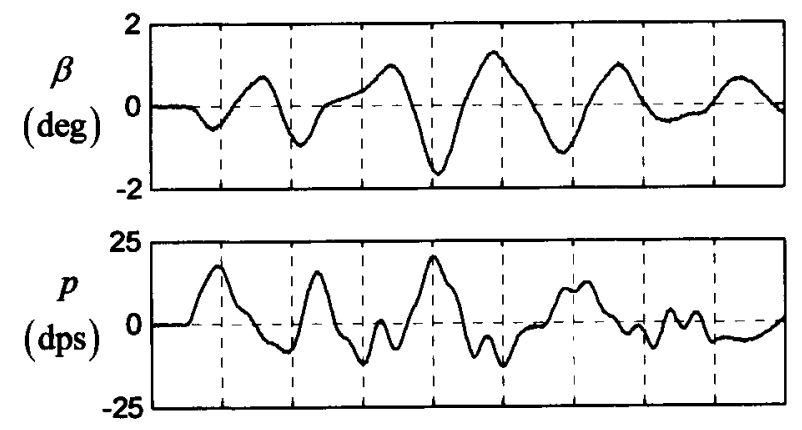

(dps)

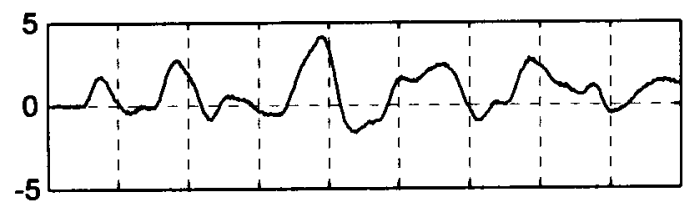

(g)

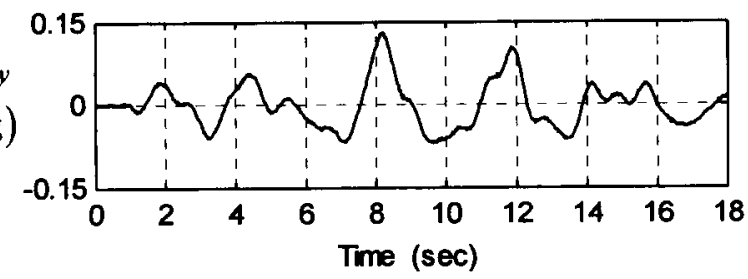

Fig. 4. Simulated F-15 ACTIVE Response
Table 1 F-15 ACTIVE Lateral/Directional Parameter Estimation Results,

$V_{o}=793 \mathrm{fps}, h_{o}=20,000 \mathrm{ft}, \alpha_{o}=\theta_{o}=2 \mathrm{deg}$

\begin{tabular}{ccccc}
\hline & $\theta_{t r u e}$ & $\hat{\theta}$ & $\begin{array}{c}\text { Std. } \\
\text { Error }\end{array}$ & $\hat{\theta}-\theta_{\text {true }}$ \\
\hline$Y_{\beta}$ & -0.150 & -0.150 & 0.0002 & -0.0001 \\
\hline$Y_{\delta_{r}}$ & 0.050 & 0.050 & 0.0003 & 0.0002 \\
\hline$Y_{\delta_{d s}}$ & 0.035 & 0.035 & 0.0003 & 0.0001 \\
\hline$Y_{\delta_{d c}}$ & -0.025 & -0.025 & 0.0003 & -0.0002 \\
\hline$L_{\beta}$ & -22.5 & -22.5 & 0.08 & 0.02 \\
\hline$L_{p}$ & -2.05 & -2.04 & 0.007 & 0.007 \\
\hline$L_{r}$ & 3.15 & 3.14 & 0.029 & -0.012 \\
\hline$L_{\delta_{a}}$ & -28.4 & -28.2 & 0.08 & 0.20 \\
\hline$L_{\delta_{r}}$ & 4.20 & 4.15 & 0.071 & -0.048 \\
\hline$L_{\delta_{d s}}$ & -34.2 & -34.1 & 0.07 & 0.17 \\
\hline$L_{\delta_{d c}}$ & 5.14 & 5.09 & 0.069 & -0.112 \\
\hline$N_{\beta}$ & 4.40 & 4.42 & 0.030 & 0.024 \\
\hline$N_{p}$ & 0.11 & 0.11 & 0.002 & 0.000 \\
\hline$N_{r}$ & -0.17 & -0.17 & 0.012 & 0.005 \\
\hline$N_{\delta_{r}}$ & -3.75 & -3.72 & 0.029 & 0.039 \\
\hline$N_{\delta_{d s}}$ & -1.40 & -1.41 & 0.029 & -0.015 \\
\hline$N_{\delta_{d c}}$ & -2.40 & -2.37 & 0.028 & 0.044 \\
\hline & & & & \\
\hline
\end{tabular}

Table 2 Multiple Input Design

\begin{tabular}{cccc}
\hline Input & $A_{k}$ & $k$ & $R P F$ \\
\hline$\delta_{a}$ & 0.707 & $3,6,9,18$ & 1.055 \\
\hline$\delta_{r}$ & 0.707 & $4,8,12,16$ & 0.995 \\
\hline$\delta_{d s}$ & 0.707 & $5,10,15,20$ & 0.995 \\
\hline$\delta_{d c}$ & 0.707 & $7,14,21$ & 1.003 \\
\hline
\end{tabular}

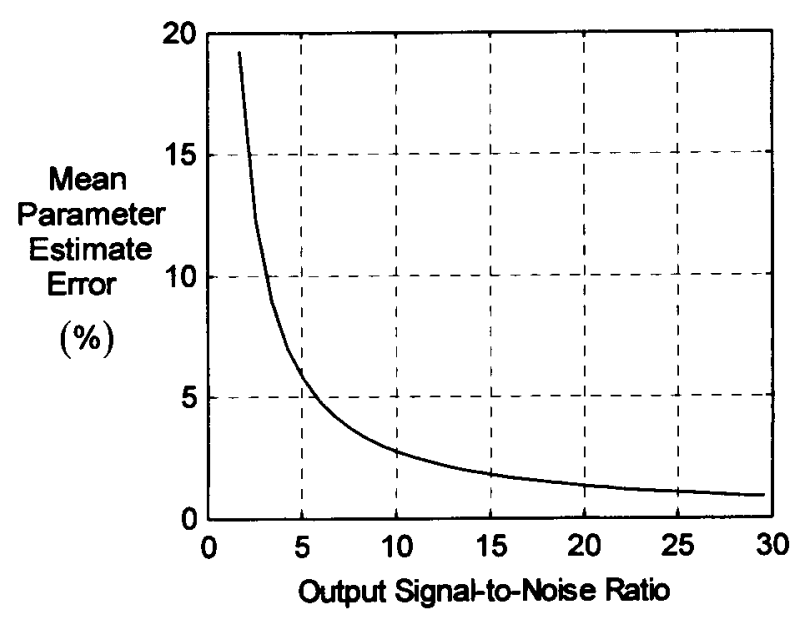

Fig. 5. Parameter Estimation Accuracy Dependence on Output Signal-to-Noise Ratio 